\title{
Was ist Tropenmedizin?
}

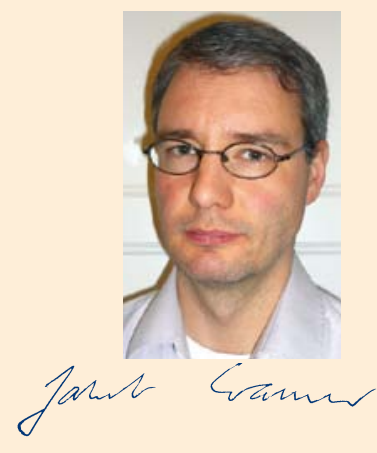

Liebe Kolleginnen und Kollegen,

Tropen- und Reisemedizin ist viel mehr als Malariabehandlung und -prophylaxe. Das wird auch in dieser Ausgabe deutlich - und gelegentlich tritt ein im weiteren Sinne tropenmedizinisches Problem im heimischen Umfeld auf, wie im Fallbericht von Florian Edsperger.

Als das Hamburger Bernhard-Nocht-Institut für Tropenmedizin im Jahr 1900 seinen Betrieb am Hamburger Hafen aufnahm, war es die Seefahrt, die mit den Handelsschiffen exotische Krankheiten nach Europa brachte. Heute erfolgt die globale Ausbreitung von Infektionen vorwiegend über die Luftfahrt. Es schleicht sich das Gefühl ein, dass neue Erreger beziehungsweise Ausbrüche und Epidemien in immer kürzeren Abständen auftreten (oder registriert werden?) - siehe Vogelgrippe H5N1 2003, Schweinegrippe H1N1 2009 und aktuell wieder ein reassortiertes Vogelgrippevirus (H7N9) in Shanghai. Ein Beitrag von Anthony Evans informiert über die internationale Notfallplanung in der Luftfahrt.

Neue Reiseziele, zunehmende Lebenserwartung und medizinischer Fortschritt führen dazu, dass immer mehr Reisende mit chronischen Grunderkrankungen ihrem Fernweh nachgeben. Wie Reisen mit COPD und Asthma, zwei der häufigsten Krankheiten weltweit, gut vorbereitet werden, erläutert Rainald Fischer.

Seismische Aktivitäten treten in beliebten tropischen/subtropischen Reisezielen vermehrt auf, auch wenn sich kaum einer am Urlaubsstrand Gedanken um Erdbeben oder Tsunamis machen dürfte. Die Übersicht von Peter Bormann veranschaulicht diese Gefahr. Sie hat im letzten Jahrzehnt wahrscheinlich mehr Opfer unter den Reisenden gefordert als die meisten impfpräventablen Infektionserkrankungen. Vielleicht wäre ein Informationsblatt dazu in der Reisesprechstunde sinnvoll.

Im tropenmedizinischen Beitrag fasst Marion Hulverscheidt die Geschichte der Gelbfieberimpfung zusammen, die - wie keine andere Impfung - mit der Tropen- und Reisemedizin verbunden ist. Sie wird oft aufgrund von regulatorischen Bestimmungen und nicht aus medizinischen Gründen verimpft und kann, trotz geringem Infektionsrisiko, Voraussetzung für eine Einreise sein. Ich möchte mich Ihnen mit diesem Editorial auch als neues Mitglied im Editorial Board vorstellen. Seit mehr als zwölf Jahren bin ich in der Tropen- und Reisemedizin tätig, zunächst in Berlin und seit 2004 in Hamburg. Insbesondere in den letzten Jahren mache ich mir Gedanken dazu, was ,Tropenmedizin' eigentlich ist oder sein sollte: Die Behandlung von Reiserückkehrern sowie die Reiseberatung beinhalten viel mehr als Infektionen. Heute sind Gesundheitsrisiken - unter anderem durch Tierkontakte, Hygiene, Intoxikationen, Umwelteinflüsse, Naturereignisse, Verkehr und Gewalt - ebenso Teil der Tropen- und Reisemedizin wie internationale Großereignisse.

Gleichzeitig scheint in den letzten Jahren in Entwicklungsländern die Relevanz von nicht infektiösen Erkrankungen wie Bluthochdruck und Diabetes stärker in den Vordergrund zu rücken. Chronische Erkrankungen gewinnen daher nicht nur bei Reisenden, sondern auch in der Bevölkerung tropischer Regionen an Bedeutung in unserem Fachgebiet. Ich möchte daher dazu anregen, sich einmal Gedanken zu ,was ist Tropenmedizin' zu machen. Ist dieser Begriff zeitgemäß? Über einen Gedankenaustausch würde ich mich freuen und vielleicht können Ihre Gedanken und Ideen zu diesem Thema in einer der kommenden Ausgaben dieser Zeitschrift aufgenommen werden. 